\title{
ARTICLE \\ Flagellin-mediated activation of IL-33-ST2 signaling by a pathobiont promotes intestinal fibrosis
}

\author{
Jin Imai ${ }^{1,2}$, Sho Kitamoto ${ }^{1}$, Kohei Sugihara ${ }^{1}$, Hiroko Nagao-Kitamoto ${ }^{1}$, Atsushi Hayashi ${ }^{1,3}$, Tina L. Morhardt ${ }^{1,4}$, Peter Kuffa ${ }^{1}$, \\ Peter D. R. Higgins ${ }^{1}$, Nicolas Barnich ${ }^{5}$ and Nobuhiko Kamada ${ }^{1}$
}

\begin{abstract}
Intestinal fibrosis is a severe complication in patients with Crohn's disease (CD). Unfortunately, the trigger leading to the development of intestinal fibrosis in the context of $C D$ remains elusive. Here, we show that colonization by a CD-associated pathobiont adherent-invasive Escherichia coli (AIEC) promotes the development of intestinal fibrosis. Exogenously inoculated AIEC strain LF82 and commensal E. coli HS were gradually eradicated from the intestine in healthy mice. In Salmonella- or dextran sodium sulfate-induced colitis models, AIEC exploited inflammation and stably colonize the gut. Consequently, persistent colonization by AIEC LF82 led to substantial fibrosis. In contrast, commensal $E$. coli HS was unable to derive a growth advantage from inflammation, thereby failing to colonize the inflamed intestine or promote intestinal fibrosis. AIEC colonization potentiated the expression of the IL-33 receptor ST2 in the intestinal epithelium, which is crucial for the development of intestinal fibrosis. The induction of ST2 by AIEC LF82 was mediated by flagellin, as the $\triangle$ fliC mutant failed to induce ST2. These observations provide novel insights into pathobiont-driven intestinal fibrosis and can lead to the development of novel therapeutic approaches for the treatment of intestinal fibrosis in the context of CD that target AIEC and/or its downstream IL-33-ST2 signaling.
\end{abstract}

Mucosal Immunology (2019) 12:632-643; https://doi.org/10.1038/s41385-019-0138-4

\section{INTRODUCTION}

Inflammatory bowel disease (IBD) is a chronic inflammatory disorder of the gastrointestinal tract, which comprises two major disease types, Crohn's disease (CD) and ulcerative colitis (UC). ${ }^{1}$ Although the precise etiology of IBD remains unknown, genetic predispositions and environmental factors, including the gut microbiota, diet, stress, smoking, cooperatively promote abnormal immune responses as well as the impairment of intestinal barrier function, thus leading to disease. ${ }^{2}$ Chronic inflammation often results in tissue fibrosis, especially in $C D$, frequently causing extensive local intestinal fibrosis and mechanical obstruction. ${ }^{3}$ Approximately $40 \%$ of CD patients with the ileal disease develop clinically apparent strictures. ${ }^{3}$ The strictures are clinically classified as fibrotic, inflammatory, or mixed types. Standard anti-inflammatory therapies, including anti-tumor necrotic factor-alpha (TNF-a) antibody treatment, can improve inflammatory strictures through the reduction of inflammation-mediated edema. On the other hand, conventional medical treatment is largely ineffective for fibrotic strictures. Hence, surgical resection and endoscopic dilation are utilized as treatment options for fibrotic strictures. Surgical resection is an option for more than $80 \%$ of stenosis patients. ${ }^{3}$ However, the post-surgery recurrence rate is close to $50 \%$ at 10 to 15 years following ileocecal resection. Likewise, the cumulative redilation-free rate after the initial balloon dilation was $64 \%$ at 2 years and $47 \%$ at 3 years. ${ }^{4}$ Thus, existing strategies for stricture management in the context of CD are insufficient and new treatment options are desperately needed.

Accumulating evidence indicates that the gut microbiota plays a pivotal role in the development and progression of IBD. The gut microbial communities in IBD patients are known to be perturbed - a condition called gut dysbiosis. ${ }^{5}$ Gut dysbiosis results in the enrichment of potentially pathogenic members of commensal bacteria (pathobionts) and/or the reduction of protective bacteria, thereby rendering the microbiota pathogenic. ${ }^{6}$ Indeed, colonization by the dysbiotic gut microbiota isolated from IBD patients is sufficient to elicit abnormal immune activation in the intestinal mucosa. ${ }^{7}$ A pathotype of Escherichia coli, namely adherentinvasive E. coli (AIEC), has been identified as a potential pathobiont associated with IBD, particularly $\mathrm{CD}{ }^{8}$ Although colonization by AIEC only results in mild inflammation in normal mice, ${ }^{9}$ AIEC causes massive intestinal inflammation in genetically susceptible hosts. Moreover, AIEC aggravates dextran sulfate sodium (DSS)induced or enteric pathogen-induced colitis. ${ }^{10,11}$ Thus, AIEC is involved in the augmentation of inflammation in the context of IBD. However, the potential role of AIEC in the development of intestinal fibrosis remains incompletely understood. In $C D$ patients, AIEC is isolated most frequently from the terminal ileum. ${ }^{8}$ Given that terminal ileum is the most common site for the formation of fibrotic stricture in $\mathrm{CD}_{1}{ }^{3}$ it is conceivable that AIEC strains target this section of the gut and contribute to the promotion of fibrosis.

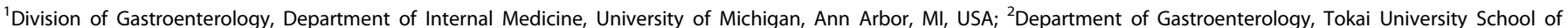

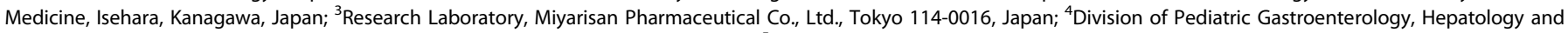

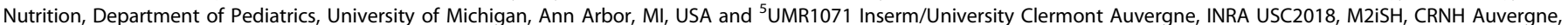
Clermont-Ferrand, France

Correspondence: Nobuhiko Kamada (nkamada@umich.edu)

Received: 31 May 2018 Revised: 17 December 2018 Accepted: 20 January 2019

Published online: 11 February 2019 


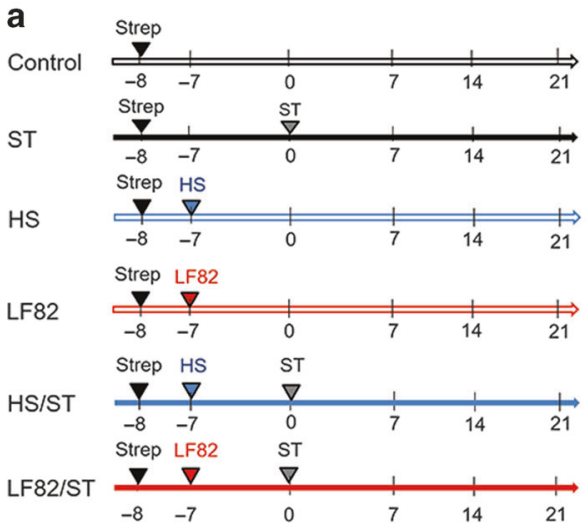

b

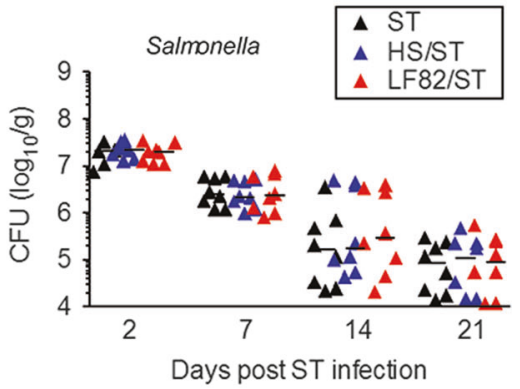

C
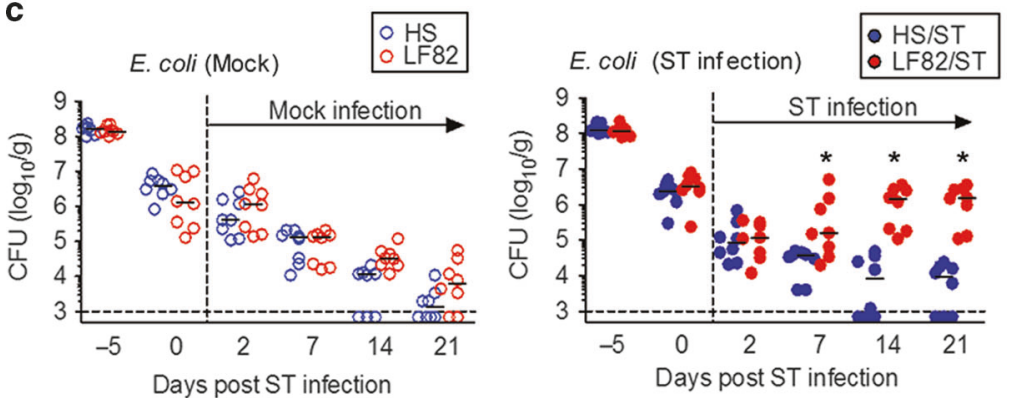

Fig. 1 AIEC colonization persists in the inflamed intestine. a SPF C57BL/6 mice were pre-treated with 20 mg of streptomycin (Strep) 1 day prior to $E$. coli inoculation. Strep-treated mice were then inoculated with human commensal $E$. coli strain HS or human AIEC strain LF82 $\left(1 \times 10^{9}\right.$ CFU). The control and ST groups only received PBS. Seven days following $E$. coli colonization, mice were infected with $2 \times 10^{7} \mathrm{CFU}$ of $S$. Typhimurium $\Delta$ spiA (ST). b, $\mathbf{c}$ Intestinal colonization (feces) of $S$. Typhimurium $\Delta$ spiA (b) and pre-colonized $E$. coli strains on indicated days post ST infection. Dots represent individual mice. Bars represent median. ${ }^{*} ; P<0.05$ by 2 -Way ANOVA with Bonferroni post-hoc test

Although an experimental model that would fully replicate the pathogenesis of intestinal fibrosis seen in IBD patients currently does not exist, various animal models have been used to study the mechanism of intestinal fibrosis associated with IBD. ${ }^{12}$ Chemicallyinduced colitis models (e.g., DSS, trinitrobenzene sulfonic acid) are widely used to model fibrogenesis in IBD. However, these substances used to induce colitis and fibrosis are toxic to the intestinal epithelium and, therefore, these models might not mimic the natural course of fibrogenesis in IBD. In addition to the chemically-induced colitis models, infection with pathogenic bacteria can be used to induce intestinal fibrosis. It has been reported that Salmonella enterica serovar Typhimurium (S. Typhimurium) infection results in the development of severe fibrosis. ${ }^{13} \mathrm{~S}$. Typhimurium infection recapitulates various pathogenic features of intestinal fibrosis seen in CD patients, such as Th1/Th17-skewed immune responses and induction of pro-fibrotic and extracellular matrix proteins. S. Typhimurium infection is, therefore, the optimal model for the investigation of immune pathways associated with fibrogenesis in CD. ${ }^{13}$ However, infection with this pathogen has not been implicated in the pathogenesis of CD. Hence, in this study, we have modified the Salmonella-induced intestinal fibrosis model and established an intestinal fibrosis model in which fibrosis is induced by the colonization of a CDassociated pathobiont AIEC. An attenuated strain of $S$. Typhimurium can cause Th1/Th17-skewed CD-like colitis, however, it is insufficient for the induction of fibrosis. ${ }^{13}$ Here, we show that cocolonization with a CD-associated pathobiont AIEC promotes the development of intestinal fibrosis in mice infected with the attenuated strain of $S$. Typhimurium. AIEC colonization likewise promotes intestinal fibrosis in the DSS model of colitis, implying that the impact of AIEC colonization on the development of fibrosis is not model specific. AIEC colonization potentiates IL-33ST2 signaling in the intestinal epithelium via production of flagellin. The AIEC-induced upregulation of ST2 is critical for the induction of fibrosis. These observations are novel and identify new avenues for the treatment of intestinal fibrosis in IBD that focus on AIEC and AIEC-mediated IL-33-ST2 signaling.

\section{RESULTS}

AIEC colonization persists in the inflamed intestine

Infection with a murine non-typhoidal Salmonella is known to model various pathogenic features of intestinal fibrosis seen in $C D$ patients and is hence widely used to study the immune pathways that are associated with fibrogenesis in CD. ${ }^{13}$ In this study, in order to address the extent to which colonization by CD-associated pathobionts contributes to intestinal fibrosis, we utilized a modified Salmonella-induced colitis and fibrosis model. A S. Typhimurium mutant strain that is deficient in type 3 secretion system encoded by the Salmonella pathogenicity island (SPI) 2 ( $\triangle$ spiA mutant) was used. This mutant strain replicates normally in the intestine but fails to disseminate systemically, thereby causing chronic colitis without killing the infected hosts. ${ }^{13}$ Importantly, SPI-2 mutant Salmonella is unable to induce the development of intestinal fibrosis. ${ }^{13}$ SPF C57BL/6 mice were pre-treated with $20 \mathrm{mg}$ of streptomycin (Strep) (Fig. 1a). After 8 days, mice were challenged with the $S$. Typhimurium $\triangle$ spiA mutant strain (ST) and Salmonella colonization was monitored until day 21 postinfection. To address the effect of AIEC colonization, mice were colonized with either a CD patient-derived AIEC strain LF82 ${ }^{8}$ or a human commensal $E$. coli strain $\mathrm{HS}^{14} 7$ days prior to Salmonella or mock infection (Fig. 1a). Salmonella colonization levels reached $>10^{7} \mathrm{CFU} / \mathrm{g}$ feces on day 2 post-infection and then gradually diminished in the gut (Fig. 1b). Colonization levels of Salmonella were not affected by the presence of $E$. coli 

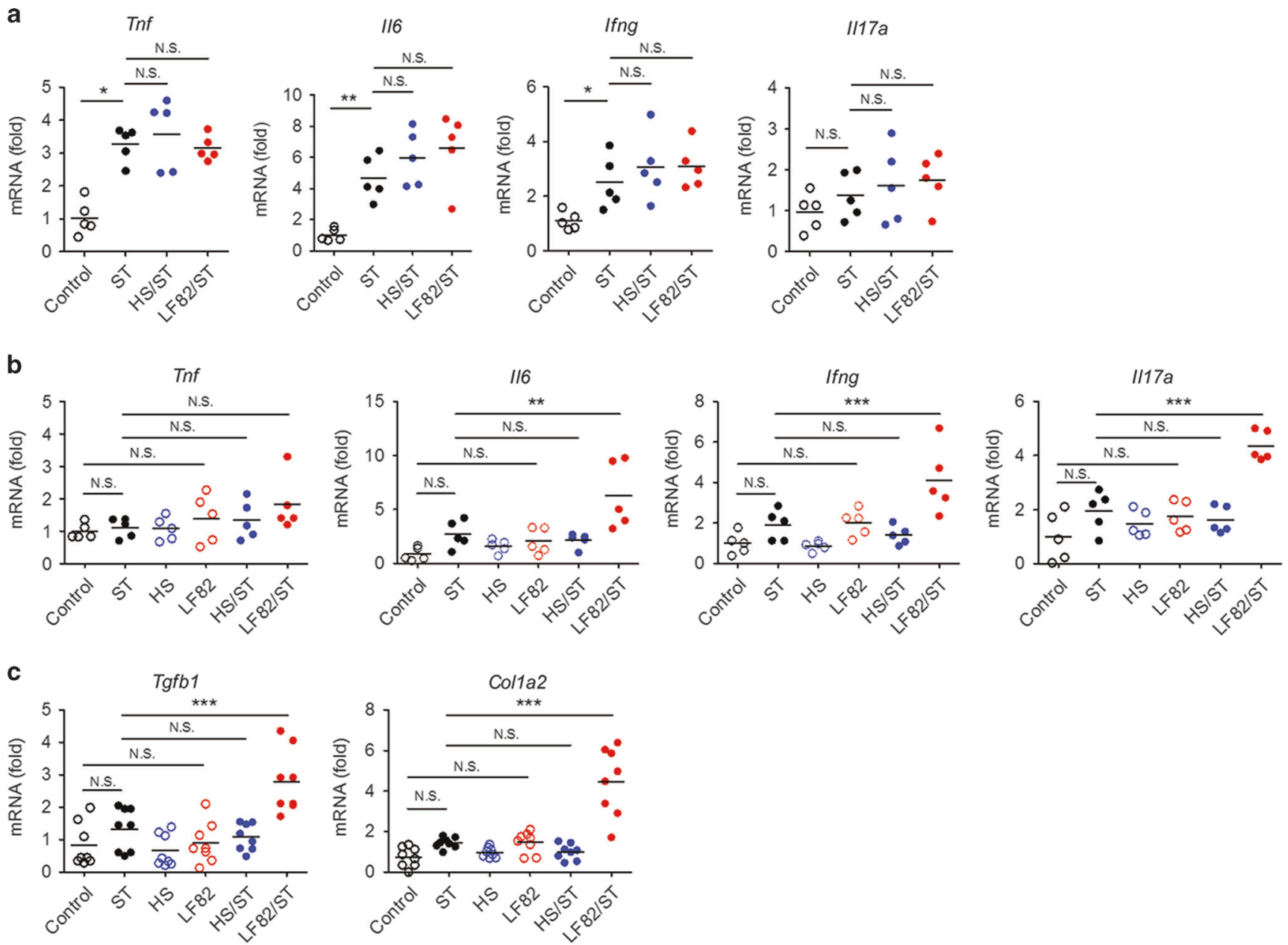

d
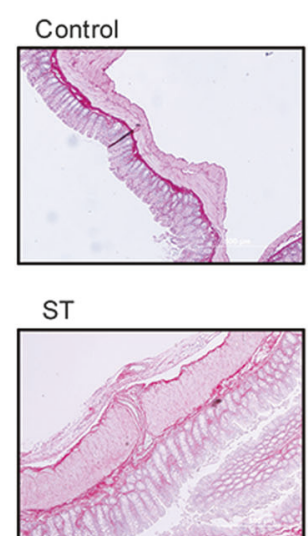

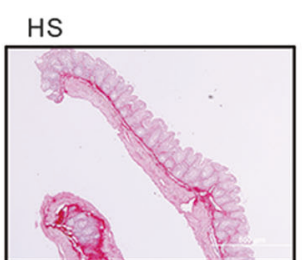

HS/ST

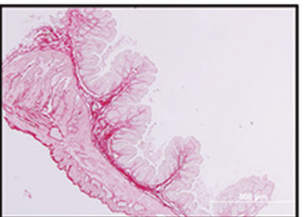

LF82

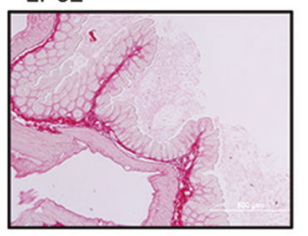

LF 82/ST

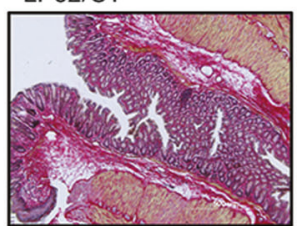

e

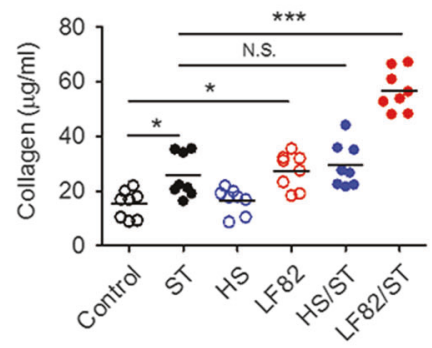

Fig. 2 AIEC colonization promotes intestinal fibrosis in Salmonella-infected mice. S. Typhimurium $\Delta$ spiA (ST) and E. coli (HS or LF82) infected mice were sacrificed on day 2 (a) and on day 21 post ST infection (b-e). Colonic tissues were harvested on day 2 (a) and on day 21 (b, c) post ST infection and mRNA expression of indicated cytokines were quantified by qPCR. The accumulation of collagen was examined by Picrosirius red staining on day 21 post ST infection (d) and collagen assay (e). Dots represent individual mice. Bars represent mean. N.S.; not significant, *; $P<0.05,{ }^{* *} ; P<0.01$, ***; $P<0.001$ by 1 -Way ANOVA with Bonferroni post-hoc test

strains (Fig. 1b). Next, we assessed the colonization potential of E. coli strains. In the absence of Salmonella (mock infection), both HS and LF82 did not stably colonize the intestine and were gradually eradicated. In contrast, in Salmonella-infected mice, only LF82 persisted on days 7, 14, and 21 post-Salmonella infection (Fig. 1C). These results suggest that AIEC, unlike commensal E. coli, is able to exploit Salmonella-caused inflammation and stably colonize the inflamed gut.
AIEC colonization promotes colonic fibrosis

Next, we assessed the effects of persistent AIEC colonization on the development of intestinal inflammation and fibrosis. In the acute phase (day 2), Salmonella-induced colitis (the expression of Tnf, II6, Ifng) was not affected by the presence of $E$. coli strains (HS or LF82) (Fig. 2a). However, in the chronic phase (day 21), the presence of LF82 amplified the expression of pro-inflammatory cytokines induced by Salmonella infection in the colonic mucosa 
a

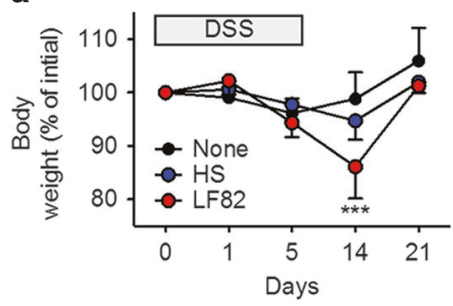

b

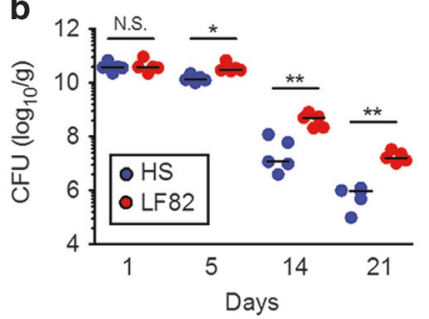

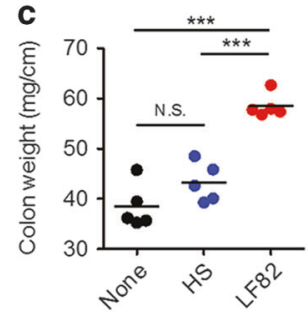

d
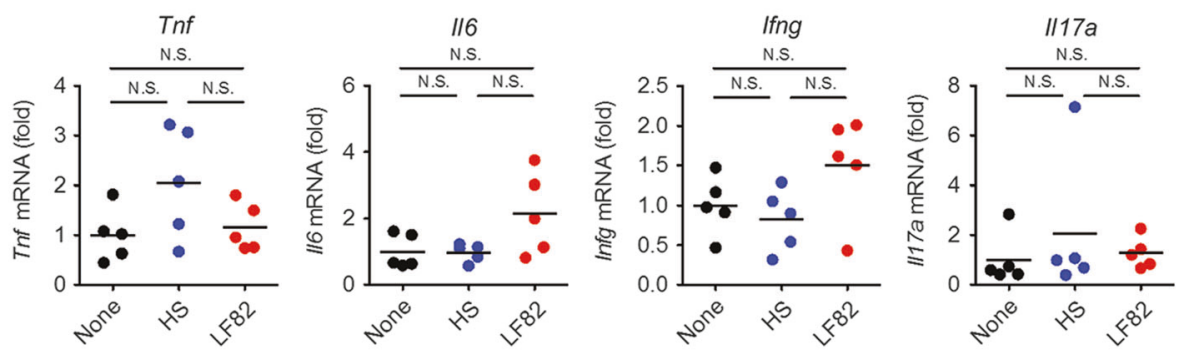

e
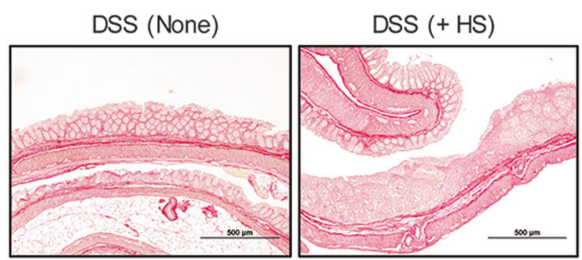

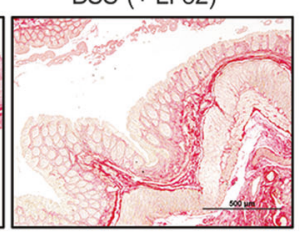

f

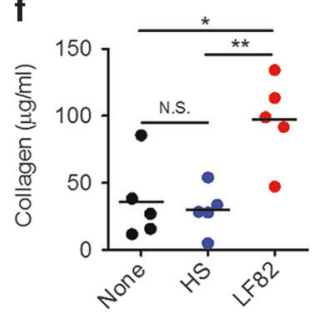

Fig. 3 AIEC colonization promotes intestinal fibrosis in DSS-induced colitis mice. SPF C57BL/6 mice were pre-treated with $20 \mathrm{mg}$ of streptomycin (Strep) 1 day prior to E. coli inoculation. Strep-treated mice were then inoculated with human commensal E. coli strain HS or human AIEC strain LF82 $\left(1 \times 10^{9} \mathrm{CFU}\right.$ each). E. coli-colonized mice were then treated with 3.0\% DSS for 5 days followed by regular water. Mice were sacrificed 21 days post DSS administration. a Body weight change. Data shown are mean \pm SD $(N=5)$. ${ }^{* * ;} P<0.001$ by 2 -Way ANOVA with Bonferroni post-hoc test (vs None). b Intestinal colonization (feces) of $E$. coli strains. Dots represent individual mice. Bars represent median. N.S.; not significant, ${ }^{*} ; P<0.05,{ }^{* *} ; \mathrm{P}<0.01$ by Mann-Whitney $U$-test. c Colon weight (per length) at day 21 . d The mRNA expression of pro-inflammatory cytokines in the colonic mucosa. e, $\mathbf{f}$ The accumulation of collagen was examined by Picrosirius red staining on day 21 (e) and collagen assay (f). c-f Dots represent individual mice. Bars represent mean. N.S.; not significant, ${ }^{*} ; P<0.05,{ }^{* *} ; P<0.01,{ }^{* * *} ; P<0.001$ by One-Way ANOVA with Bonferroni post-hoc test

(Fig. 2b). In contrast, E. coli HS did not influence the severity of colitis caused by Salmonella, even in the chronic phase (Fig. 2b). Next, we examined colonic fibrosis to further assess the impact of AIEC colonization on Salmonella-induced chronic inflammation. Fibrosis was evaluated by assessing the expression of fibrosisrelated factors Tgfb 1 and Col1a2 in the colonic mucosa, Picro-sirius red staining and quantifying the amount of collagen deposited in the colonic tissues. Consistent with a previous report, ${ }^{13}$ infection with the mutant S. Typhimurium deficient in SPI-2 $(\triangle$ spiA) did not upregulate fibrosis-related genes or increase collagen deposition (Fig. 2c-e). Notably, upregulation of fibrosis genes and a massive deposition of collagen in the extracellular matrix (ECM), especially in the submucosal layer, were observed in the colonic mucosa of Salmonella/AIEC co-infected group (ST/LF82) (Fig. 2c-e). In contrast, HS colonization or Salmonella /HS co-colonization did not promote colonic fibrosis (Fig. 2c-e). These results indicate that persistent AIEC colonization results in chronic inflammation and fibrosis in the colon.

\section{AIEC colonization promotes intestinal fibrosis in DSS-induced} colitis

Next, we examined the impact of AIEC colonization on the development of intestinal fibrosis in another IBD model. SPF C57BL/6 mice were pre-treated with $20 \mathrm{mg}$ of streptomycin 1 day prior to AIEC LF82 or commensal E. coli HS colonization. E. colicolonized mice were then treated with $3.0 \%$ DSS for 5 days followed by regular water until day 21 (Fig. 3a). Commensal E. coli
HS colonization did not affect body weight loss in DSS-treated mice (Fig. 3a). In contrast, mice colonized by AIEC LF82 lost significantly more body weight on day 14 compared to control mice not colonized by exogenous E. coli (none) (Fig. 3a). Consistent with the Salmonella-induced colitis model, LF82 colonized more efficiently than HS in DSS-treated mice during inflammation (Fig. 3b). Moreover, the colon tissue isolated from DSS-treated mice colonized by AIEC was significantly thicker than that isolated from DSS-treated mice colonized by HS or control DSS-treated mice not colonized by exogenous E. coli (Fig. 3c). However, colonic inflammation was not significantly enhanced by AIEC colonization (Fig. 3d). This evidence suggests that colon thickening in AIEC-colonized mice may not result from increased levels of inflammation. Rather, it is conceivable that AIEC colonization promoted colonic fibrosis in this model. Consistent with this notion, massive deposits of collagen were observed in the colonic tissue of DSS-treated mice colonized by LF82 compared to those colonized by HS or without $E$. coli colonization (Fig. $3 e, f)$. These results suggest that, in addition to the colitis induced by a microbial pathogen, persistent AIEC colonization leads to the development of fibrosis in the colon in a chemicallyinduced colitis model.

AIEC does not directly promote collagen deposition in fibroblasts To unravel how AIEC colonization promotes the development of fibrosis, we first tested whether AIEC colonization directly initiates collagen deposition in fibroblasts, which play a central role in 

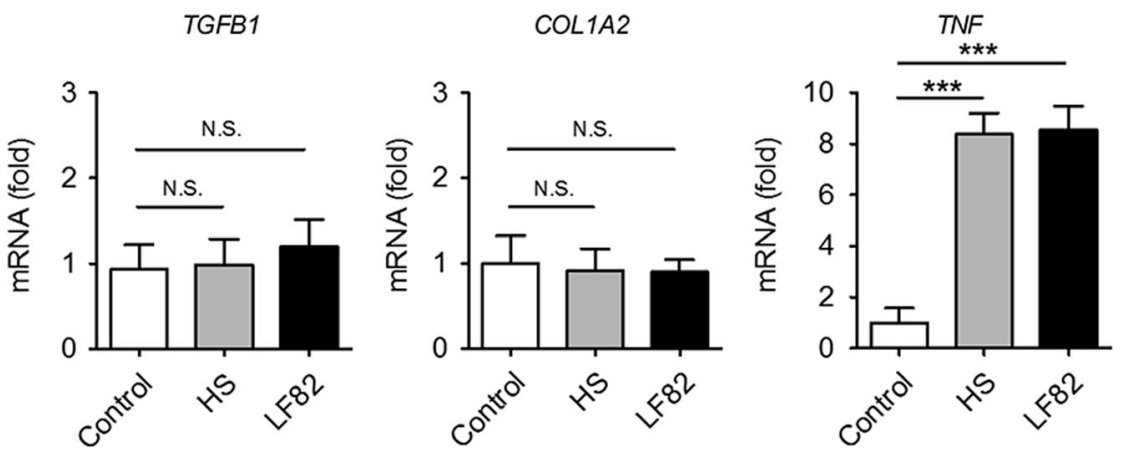

Fig. 4 AIEC does not directly promote collagen deposition in colonic fibroblasts. Human primary colonic fibroblasts $\left(1 \times 10^{4}\right.$ cells $/ 500 \mu l /$ well; 24 well plate) were stimulated with $E$. coli HS or LF82 at MOI $=10$ for $3 \mathrm{~h}$ followed by $15 \mathrm{~h}$ of additional culture in the presence of gentamycin $(100 \mu \mathrm{g} / \mathrm{ml})$ to prevent bacterial overgrowth. The expression of fibrosis-related mediators and a pro-inflammatory cytokine was quantified by qPCR. Data shown are mean \pm SD $(N=6) .{ }^{*} ; P<0.05$ by One-Way ANOVA with Bonferroni post-hoc test

organ fibrogenesis. We stimulated human primary colonic fibroblasts with LF82 or HS, and then measured the expression of fibrosis-related factors TGFB1 and COL1A2. As shown in Fig. 4, stimulation with neither LF82 nor HS elicited the expression of TGFB1 and COL1A2 in human fibroblasts. In contrast, stimulation with $E$. coli significantly upregulated the expression of proinflammatory cytokine TNF (Fig. 4). These results suggest that AIEC does not directly induce fibrotic programs in fibroblasts, although these cells can sense the bacterium and elicit inflammatory responses.

AIEC colonization promotes intestinal fibrosis via the activation of IL-33-ST2 signaling

IL-33, an IL-1 family cytokine, is implicated in the pathogenesis of fibrotic diseases in various organs. ${ }^{15}$ IL-33 binds to its receptor ST2, leading to the activation of nuclear factor-kB (NF-kB) and mitogenactivated protein kinases (MAPKs) that cooperatively drive the production of pro-inflammatory and type 2 helper T cell (Th2)associated cytokines. ${ }^{15}$ Th2 cytokines, especially IL-13, promote TGF- $\beta$ production and collagen deposition in the ECM. ${ }^{16}$ In our model, mono-colonization with Salmonella or E. coli strains (commensal HS or AIEC LF82) did not induce the expression of II33 and I/1r/1 (gene encodes ST2) (Fig. 5a). In contrast, cocolonization with Salmonella and AIEC (group ST/LF82) resulted in a marked upregulation of $\| / 33$ and $/ 11 r \| 1$ in the colon, while HS colonization did not augment the expression of these genes in Salmonella-infected animals (HS/ST group) (Fig. 5a). Moreover, AIEC LF82 colonization, unlike commensal $E$. coli HS, induced the expression of $\| 11 \mathrm{rl}$ in the DSS model of colitis (Fig. 5b). Next, we validated these results in human CD patients. Using a publically available dataset (GSE75214), ${ }^{17}$ we discovered that the expression of IL-33 (IL33) and ST2 (ILIRL1) are significantly upregulated in the ileal mucosa of active CD patients compared to that of normal controls or inactive CD patients (Fig. $5 \mathrm{C}$ ). To assess the role of IL-33ST2 signaling in AIEC-elicited intestinal fibrosis, we attempted to block this pathway during AIEC and Salmonella co-infection. Streptomycin-treated mice were colonized with LF82 for 7 days, followed by Salmonella infection for 21 days. The anti-ST2 blocking antibody or an isotype control lgG was injected intraperitoneally 3 times a week starting from day 7 post-Salmonella infection (Fig. $5 \mathrm{~d}$ ). Salmonella and E. coli (LF82) colonization was not influenced by blockade of IL-33-ST2 signaling (Fig. 5e). Likewise, blockade of IL33-ST2 signaling did not affect the expression of pro-inflammatory cytokines, such as TNF- $a$, IL-6, IFN- $\gamma$, and IL-17A (Fig. 5f). In contrast, IL-33-ST2 signaling blockade suppressed AIEC colonizationinduced upregulation of fibrosis-related genes in the colonic mucosa (Fig. 5g). Consistently, diminished IL-33-ST2 signaling significantly attenuated the accumulation of collagen in the colon (Fig. $5 \mathrm{~h}, \mathrm{i}$ ). These data suggest that AIEC colonization promotes intestinal fibrosis via the activation of IL-33-ST2 signaling.
CD-associated $E$. coli strains induce ST2 expression in IECS The IL-33 receptor ST2 is known to be expressed in various immune and non-immune cells. In the gastrointestinal tract, it is reported that intestinal epithelial cells (IECs) express high levels of ST2. ${ }^{18}$ Likewise, IECs are major producers of its ligand IL-33. ${ }^{19}$ Hence, we asked whether AIEC colonization can elicit the expression of ST2 and/or IL-33 in IECs. To this end, T84 cells, a human colonocyte cell line, were stimulated with $E$. coli HS or LF82 and the expression of IL-33 and ST2 was assessed by qPCR. As a control, human primary colonic fibroblasts were also stimulated with $E$. coli strains. As expected, only stimulation with AIEC LF82 elicited a marked upregulation of IL33 (IL-33) and IL1RL1 (ST2) expression (Fig. 6a). Neither E. coli strains induced the expression of these genes in fibroblasts (Fig. 6a). Next, we confirmed this result in vivo. To this end, we examined the expression of ST2 in the colonic mucosa of Salmonella-infected and Salmonella/E. coli co-infected mice (Fig. 6b). Consistent with the in vitro result, coinfection with Salmonella and AIEC (LF82/ST) resulted in the expression of ST2 protein in the colonic epithelium (Fig. 6b), whereas mono-infection with Salmonella (ST) or co-infection with Salmonella and E. coli HS (HS/ST) did not cause ST2 expression in the colonic mucosa (Fig. $6 \mathrm{~b}$ ). These results suggest that the CDassociated $E$. coli strain induces ST2 expression in the colonic epithelium.

Flagellin mediates the induction of ST2

Since one of AIEC's pathogenic features is its ability to adhere to and invade IECs, ${ }^{8}$ we next assessed whether epithelial adhesion/ invasion trigger the expression of these genes. To this end, we utilized a panel of AIEC and non-AIEC strains isolated from CD patients (except HS) and used them to stimulate IECs. Consistent with the results obtained from LF82, all tested AIEC strains (strain LF16, LF31, LF73, and LF82) elicited the expression of IL 1RL1 in IECs (Fig. 7a). The non-AIEC strains, with the exception of LF1, were not able to induce ILIRL1 (Fig. 7a). Although two AIEC strains (LF82 and LF73) were able to induce the expression of IL33, all the other AIEC and non-AIEC strains failed to upregulate IL33 expression (Fig. 7a). These results suggest that the ability of $E$. coli to adhere/ invade to IECs is not required for the induction of ST2. Notably, IL33 induction was not induced by most of CD-associated E. coli strains, indicating that the induction of ST2, rather than its ligand IL-33, might be a key fibrosis-initiating event resulting from pathobiont colonization in the context of CD. Next, we sought to identify the microbial factor(s), present in the CD-associated pathobiont LF82, involved in the induction of ST2. We employed the isogenic mutant strains of LF82 that lack FimH (adhesin), ${ }^{20} \mathrm{FliC}$ (flagellin synthesis), ${ }^{21}$ OmpA or OmpC (outer membrane proteins). ${ }^{22}$ T84 cells were stimulated with WT LF82 or the mutant strains, and the expression of ILIRLI and IL33 was assessed by qPCR. The LF82 $\triangle$ fimH mutant induced ILIRL1 at levels similar to 
a

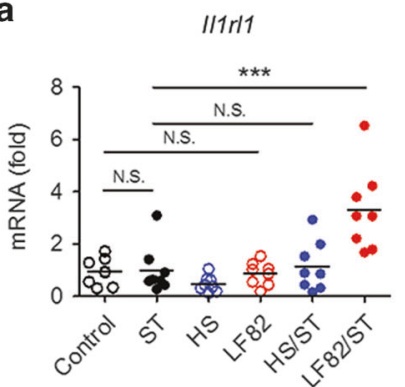

C

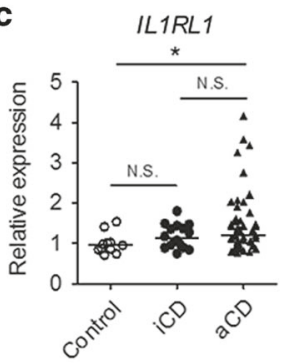

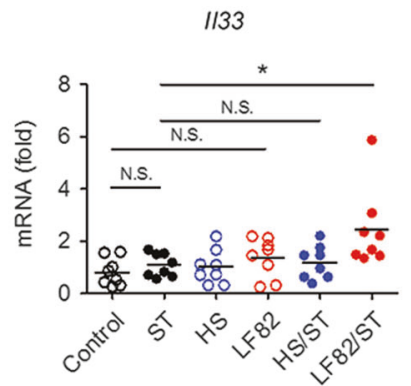

b

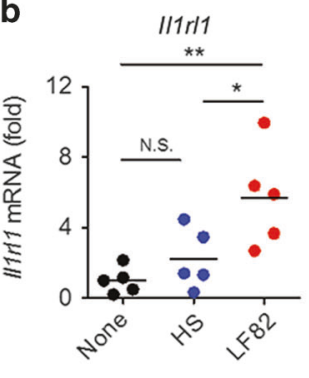

d

Control Ab

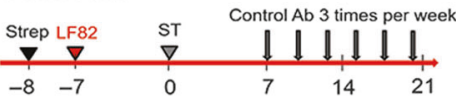

\section{$a \mathrm{ST} 2 \mathrm{Ab}$}

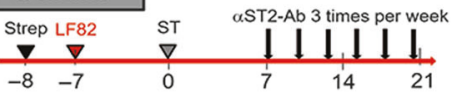

e
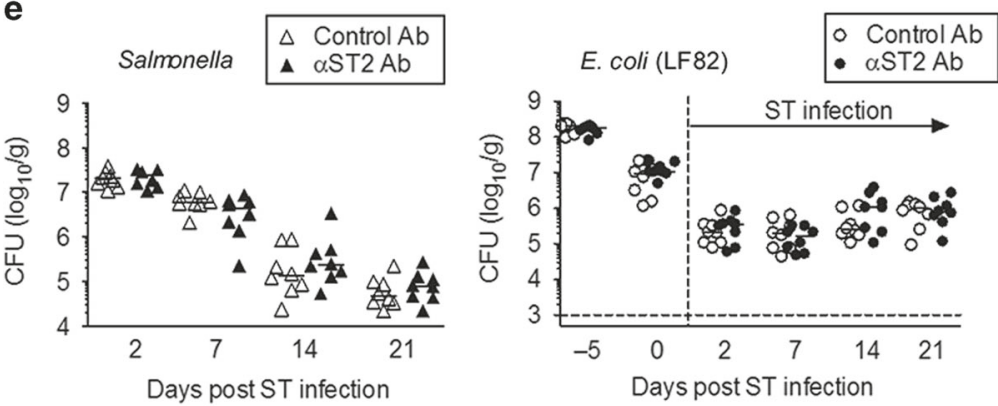

f
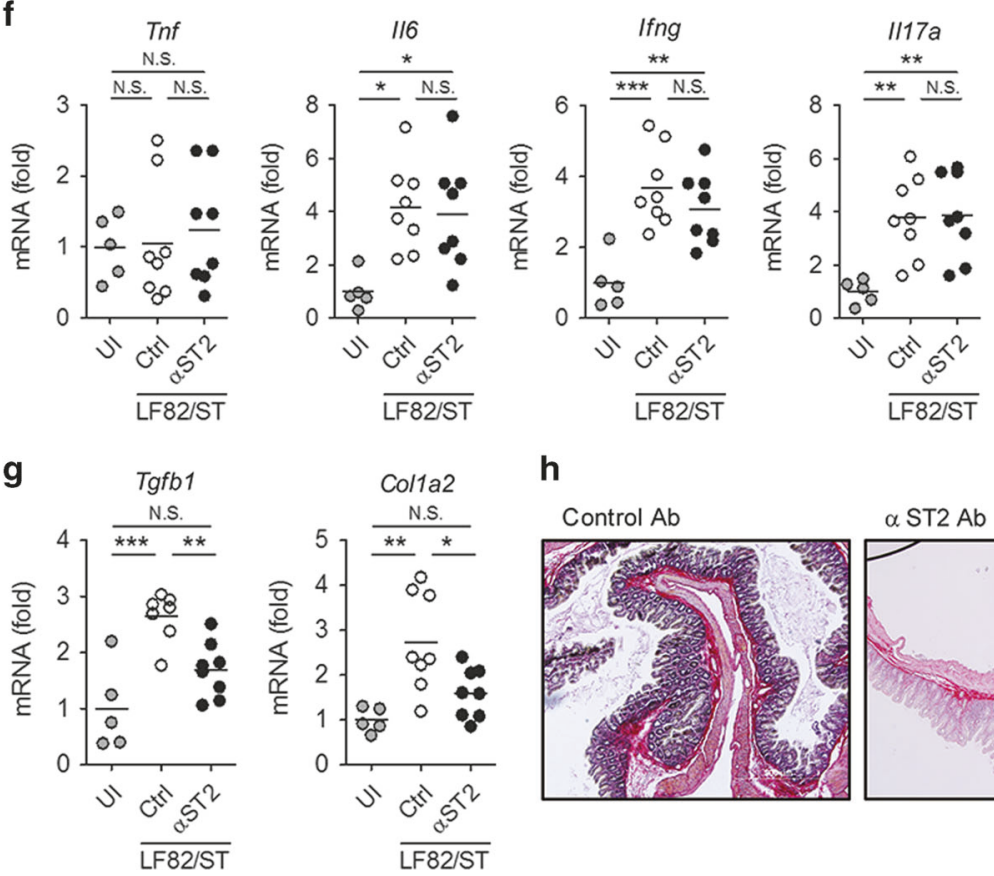

h
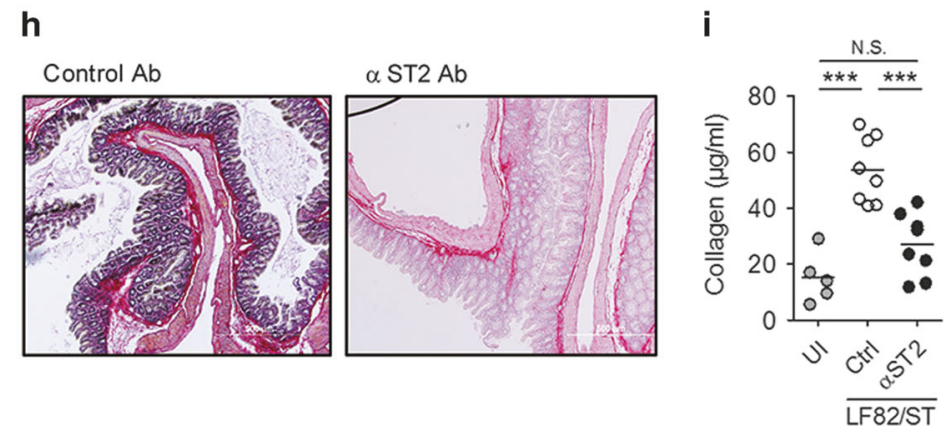

WT LF82, indicating that epithelial adhesion is not required for this process (Fig. $7 b$ ). Similarly, the $\triangle o m p A$ and $\triangle o m p C$ mutants were able to induce the expression of ILIRL1. Notably, the LF82 $\triangle$ fliC mutant was significantly impaired in its ability to induce ILIRL1, suggesting that flagellin is a key bacterial factor driving the expression of ST2 in IECs. Interestingly, all tested mutant strains, including the LF82 $\triangle$ fliC mutant, induced IL-33 with equal efficiency as their parental strain WT LF82. 
Fig. 5 AIEC colonization promotes intestinal fibrosis via the activation of IL-33-ST2 signaling. a, b The mRNA expression of IL-33 (II33) and its receptor ST2 (I/1r/1) in the colonic mucosa Salmonella/E.coli co-colonization (day 21) (a) and DSS + E. coli (day 21) (b) models. c mRNA expression of ST2 (IL1RL1) and IL-33 (IL33) genes in the ileal tissue from control subjects $(N=11)$, patients with inactive CD $(N=16)$ and active $\mathrm{CD}(\mathrm{N}=51)$. Data were derived from Gene Expression Omnibus (GEO) dataset GSE75214. Bars represent median. N.S.; not significant, *; $P<$ 0.05 , **; $P<0.01,{ }^{* * *} ; P<0.001$ by Kruskal-Wallis test. d Mice pre-colonized with AIEC strain LF82 were infected with S. Typhimurium $\Delta$ spiA (ST). Anti-ST2 blocking antibody and isotype control lgG (50 $\mu \mathrm{g} / \mathrm{mouse})$ were injected intraperitoneally 3 times per week starting on day 7 postSalmonella infection (6 times total). Mice were sacrificed 21 days following ST infection. e Intestinal colonization (feces) of S. Typhimurium $\triangle$ spiA (left) and pre-colonized E. coli LF82 (right) on indicated days following ST infection. $\mathbf{f}$ mRNA expression of indicated cytokines 21 days post ST infection. $\mathbf{g}$ Expression of fibrosis-related genes in the colonic mucosa (day 21). $\mathbf{h}, \mathbf{i}$ Accumulation of collagen was quantified by Picrosirius red staining (h) and collagen assay (i). Dots represent individual mice. Bars represent median (e) or mean (f, g, i). N.S.; not significant, ${ }^{*}$; $P<0.05,{ }^{* *} ; P<0.01,{ }^{* * *} ; P<0.001$ by One-Way ANOVA with Bonferroni post-hoc test

To identify the receptor involved in AIEC-mediated ST2 expression in IECs, we next knocked-down the known flagellin receptors TLR5 and NLRC4 in IECs. The introduction of siRNA significantly reduced the expression of these receptors in T84 cells (Fig. 7c). Our results showed that both TLR5 and NLRC4 are involved in the induction of ST2 by AIEC (Fig. 7d). To validate the role of flagellin in the induction of intestinal fibrosis in the attenuated Salmonella/AIEC co-infection model, we next coinfected streptomycin-treated mice with the SPI-2 mutant of Salmonella and WT or the $\triangle$ fliC mutant of LF82 (Fig. 8a). Flagellin production by AIEC did not affect the ability of Salmonella to colonize the intestine and Salmonella was eliminated from the mice in both WT LF82/ST and $\triangle$ fliC LF82/ST groups with the same efficiency (Fig. 8b). Likewise, the WT and $\Delta$ fliC LF82 strains colonized equally well in the inflamed gut, indicating that flagellin is not required for adaptation to the inflammatory microenvironment (Fig. 8b). The expression of pro-inflammatory cytokines in the colonic mucosa was not attenuated in the $\Delta$ fliC LF82/ST group compared to the WT LF82/ST group (Fig. 8C), suggesting that LF82 is able to augment Salmonella-induced inflammation in the absence of flagellin secretion. However, the $\triangle$ fliC mutant LF82 did not induce the expression of ST2 in the colonic mucosa (Fig. 8d, e). As a result of impaired ST2 induction, the expression of fibrosis-related genes was significantly reduced in the $\Delta$ fliC LF82/ ST group compared to the WT LF82/ST group (Fig. 8f), thereby leading to the attenuated accumulation of collagen in the colon (Fig. $8 \mathrm{~g}, \mathrm{~h}$ ). These data suggest that AIEC's flagellin promotes intestinal fibrosis.

\section{DISCUSSION}

It has become evident that the gut microbiota in IBD patients is significantly perturbed compared to non-IBD subjects. ${ }^{5}$ However, the impact of gut dysbiosis on disease outcomes remains poorly understood. A recent report has demonstrated that colonization of mice with dysbiotic microbiotas obtained from $C D$ patients sufficiently recapitulates the pro-inflammatory responses seen in CD patients. ${ }^{7}$ Thus, dysbiosis in IBD might be accompanied by an abnormal accumulation of pathobionts whose colonization likely elicits intestinal inflammation. However, the identities of IBDassociated pathobionts remain largely unknown. AIEC is an E. coli pathotype found to be more prevalent in the gastrointestinal tract of patients with $C D{ }^{23}$ As of now, the consequences of chronic colonization by AIEC in CD patients are incompletely understood.

In the present study, we have demonstrated that persistent gut colonization by the AIEC strain LF82 potentiated the development of intestinal fibrosis in Salmonella-induced colitis model in mice. Interestingly, colonization by a human commensal $E$. coli strain HS (a symbiont strain) did not affect intestinal fibrosis, indicating that induction of fibrosis is a specific feature of AIEC. Notably, neither pathobiont nor symbiont $E$. coli strains influenced intestinal inflammation caused by Salmonella infection, suggesting that promotion of fibrosis, resulting from AIEC colonization, cannot be explained by considering AIEC-triggered inflammation alone. One possible explanation is that AIEC adapts better to intestinal inflammation compared to the commensal E. coli strain and an increased abundance of $E$. coli might lead to intestinal fibrosis regardless of AIEC signatures. In this context, we have found that Salmonella-induced intestinal inflammation facilitated LF82 colonization, while not affecting HS. Neither $E$. coli strain was able to stably colonize the intestine in the absence of Salmonella. In IBD patients, it is well documented that Enterobacteriaceae (phylum Proteobacteria) are over-represented in the gut microbiota. ${ }^{5}$ In general, a healthy gut microbiota consists of obligate anaerobes, such as Clostridiales and Bacteroidales, while bacteria belonging to the family Enterobacteriaceae, including E. coli, are only minor constitutes. On the other hand, intestinal inflammation tends to foster blooms of Enterobacteriaceae at the expense of obligate anaerobes. Intestinal inflammation elicits the infiltration of inflammatory immune cells in the mucosa, such as neutrophils. These immune cells express oxidative enzymes (e.g., iNOS, NOX1, DUOX2, PHOX, SOD, and MPO) capable of generating antimicrobial radicals, such as superoxide, peroxide, hypochloride, nitric oxide, and peroxynitrite. Further oxidation of these molecules yields nitrates, $\mathrm{N}$-oxides, S-oxides and other compounds that serve as electron acceptors. Facultative anaerobes, including Enterobacteriaceae, rely on these products to utilize anaerobic respiration in the intestine. ${ }^{24,25}$ Since both AIEC and commensal E. coli can utilize anaerobic respiration, it is conceivable that AIEC employs specific, as yet unknown, mechanisms that allow it to adapt to the inflammatory environment with greater efficiency. Further study is needed to unravel the unique metabolic pathways utilized by AIEC to adapt to the inflammatory environment.

In this study, we have demonstrated that AIEC colonization elicits expression of the IL-33 receptor ST2 in IECs, which in turn augments IL-33 signaling and promotes the development of intestinal fibrosis. IL-33 is a member of the IL-1 cytokine family that has been discovered relatively recently. ${ }^{15}$ IL-33 engages its receptor ST2 expressed on various immune and non-immune cells, and activates its downstream signaling. ${ }^{15}$ The IL-33-ST2 signaling pathway is considered to be crucial for type 2 immune responses since it induces the production of IL-5 and IL-13 in Th2 cells and/or granulocytes. IL-33 is a protective cytokine important for promotion of wound healing. ${ }^{15}$ However, excessive tissue regeneration mediated by IL-33 can lead to the development of fibrosis in various organs, such as liver cirrhosis, systemic sclerosis and pulmonary fibrosis. ${ }^{26-28}$ Thus, the role of IL-33-ST2 in IBD remains controversial. Experimental animal IBD models have been used to demonstrate that IL-33-ST2 signaling promotes intestinal inflammation via enhancing IL-4. ${ }^{29}$ On the other hand, mice deficient in ST2 develop more severe colitis than control mice in DSS-induced and TNBS-induced IBD models, indicating that IL-33ST2 signaling has a protective role. ${ }^{18}$ Likewise, a recent study has also reported that IL-33 aids tissue healing in the gut by enhancing the differentiation of goblet cells and M2 type macrophages, thus protecting mice from DSS and TNBS-induced colitis. ${ }^{18}$ This discrepancy might in part be due to variability in the genetic backgrounds of experimental animals. The former study utilized Balb/c (Th2 skewed) animals whereas C57BL/6 (Th1 skewed) animals were used in the latter two studies. This 
a

IEC
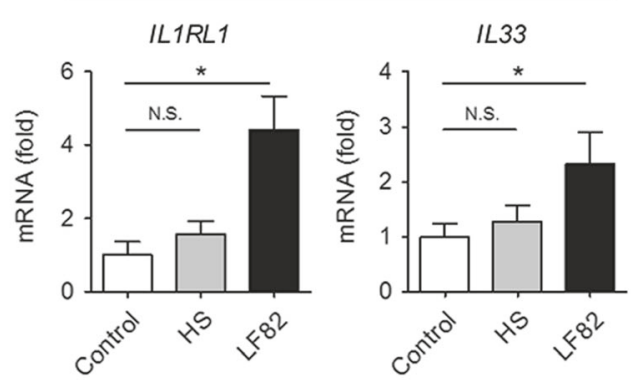

ST
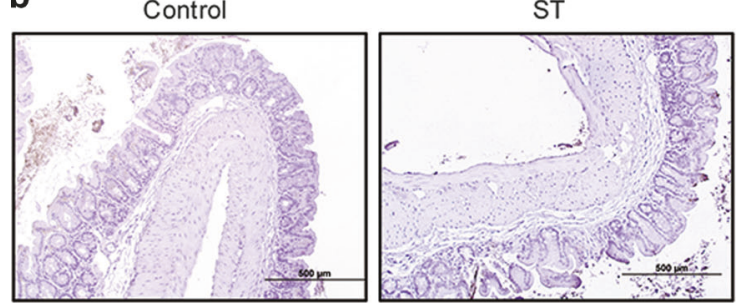

Fibroblast

IL1RL1
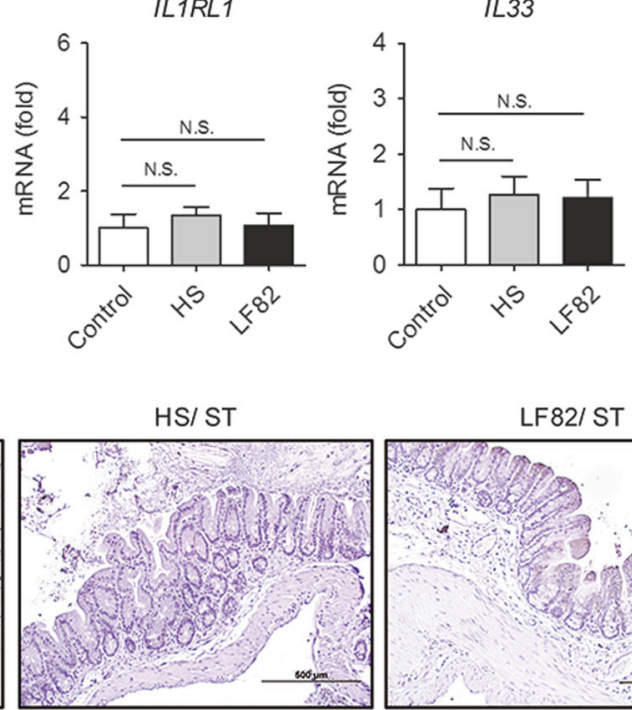

LF82/ ST

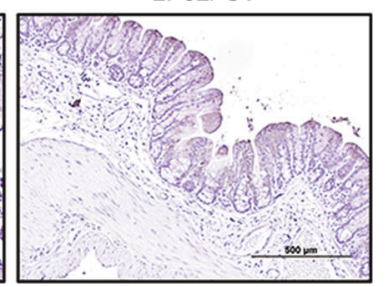

Fig. 6 CD-associated E. coli strains induce ST2 expression in IECs. a Human IEC T84 cells and primary colonic fibroblasts $\left(1 \times 10^{5}\right.$ cells/500 $\mu$ l/ well; 24 well plate) were stimulated with E. coli HS or LF82 at MOI = 10 for $3 \mathrm{~h}$ followed by $15 \mathrm{~h}$ of additional culture in the presence of gentamycin $(100 \mu \mathrm{g} / \mathrm{ml})$ to prevent bacterial overgrowth. mRNA expression of IL-33 (IL33) and ST2 (IL1RL1) was quantified by qPCR. Data shown are mean \pm SD $(N=6)$. N.S.; not significant, ${ }^{*} ; P<0.05$ by One-Way ANOVA with Bonferroni post-hoc test. $\mathbf{b}$ Strep-treated mice were then inoculated with human commensal E. coli strain HS or human AIEC strain LF82 $\left(1 \times 10^{9} \mathrm{CFU}\right)$. The control and ST groups only received PBS. Seven days following $E$. coli colonization, mice were infected with $2 \times 10^{7}$ CFU of S. Typhimurium $\Delta$ spiA (ST). At day 21 post ST infection, the colonic tissues were harvested and ST2 was strained. Representative images of ST2 immunohistochemistry are shown

may indicate that the IL-33-ST2 pathway plays different roles in UC and $C D$, as these two disease forms are associated with differently skewed adaptive immunity. ${ }^{1}$ In human IBD, IL-33 expression is reported to be elevated in the inflamed mucosa, mainly in UC and, to a lesser extent, in CD. ${ }^{30,31}$ In contrast, blood levels of IL-33 are found to decrease only in UC patients as compared to healthy control subjects. ${ }^{32}$ Additionally, blood levels of soluble ST2 (sST2), which antagonizes the activity of IL-33, are elevated in both UC and $C D .^{32}$ In this study, we analyzed the publically available GEO dataset to discover that the expression of both IL-33 and ST2 is upregulated in the ileal mucosa of patients with active $C D$, although this analysis cannot discriminate between soluble and membrane-bound ST2, which play opposite roles with respect to IL-33 signaling.

Various cell types are known to express membrane-bound ST2. ST2 is involved in IL-33 signaling and is primarily expressed, under normal conditions, in IECs in both mice and humans. ${ }^{18}$ During intestinal inflammation, ST2-positive cells can also be found in subepithelial infiltrates in both murine colitis models as well as human IBD patients. ${ }^{18}$ Therefore, it is possible that other ST2expressing cells also contribute to AIEC-induced intestinal fibrosis. Recent accumulating evidence suggests that group 2 innate lymphoid cells (ILC2), known to express high levels of ST2, play a crucial role in the development of fibrosis via activating Th2 immune responses, as demonstrated in various models of fibrosis, such as liver and lung. ${ }^{26,33}$ However, a recent report has shown ILC2 to be dispensable for the development of intestinal fibrosis. ${ }^{34}$ Thus, ILC2 might not be involved in AIEC-induced intestinal fibrosis.

In this study, we have been able to demonstrate that AIEC colonization enhances ST2 expression in IECs, which in turn augments the sensing of IL-33 in the intestine. However, the identity of pathways downstream of IL-33-ST2 that leads to fibrosis remains elusive. Since the blocking of ST2 resulted in a decreased expression of TGF- $\beta$ and collagen in the intestine, AIECmediated activation of the IL-33-ST2 pathway might eventually lead to the upregulation of TGF- $\beta$, thereby promoting collagen deposition in fibroblasts. In the lung, pulmonary epithelial cells express TGF- $\beta .^{35}$ Thus, it is possible that IECs produce TGF- $\beta$ in response to IL-33 and promote collagen expression in fibroblasts through mutual interaction. Another possibility is that IL-33 signaling in IECs alters the function of IECs, which in turn induce the activation and/or differentiation of other TGF- $\beta$ producing cells, such as macrophage/dendritic cells (DCs). For example, thymic stromal lymphoprotein (TSLP), which is induced by IL-33, is known to condition DCs and skew them toward the type 2 phenotype, including upregulation of TGF- $\beta$ expression. ${ }^{36}$ It follows, then, that TSLP conditioned-DCs might mediate profibrotic responses. Further research is required to identify the downstream mediators that link IL-33 signaling and intestinal fibrosis.

Our present results indicate that flagellin, a principal component of bacterial flagella, is a key molecule produced by AIEC that activates the expression of ST2 in IECs and subsequently causes the development of fibrosis. The role of bacterial flagella in locomotion is well documented. ${ }^{37}$ A decrease in the expression of type 1 pili, required for cell adhesion, has recently been observed in an isogenic mutant of LF82 that lacks fliC, the gene that encodes flagellin. ${ }^{37}$ As a result, the fitness and virulence of the LF82 $\triangle$ fliC mutant were significantly impaired both in vitro and in vivo. $^{37}$ In our study, the ST2 inducing capacity of AIEC LF82 remained intact when the fimH gene, which encodes adhesin and thus facilitates AIEC adhesion to IECs, was mutated. Consistently, a non-AIEC strain LF1, which cannot adhere/invade IECs, was also capable of inducing the expression of ST2 in IECs. It has been reported that TLR5 plays an important role in the sensing of AIEC. ${ }^{38}$ Consistently, we found that AIEC colonization induces ST2 expression in IECs through TLR5. In addition to TLR5, our result indicates that the activation of NLRC4 signaling by flagellin may also be used for the induction of ST2.

Our study has demonstrated that flagellin, produced by AIEC, is critical for the induction of ST2 expression in IECs. However, it remains unclear why only AIEC induces ST2 and promotes intestinal fibrosis; after all, both AIEC and commensal E. coli HS 
a

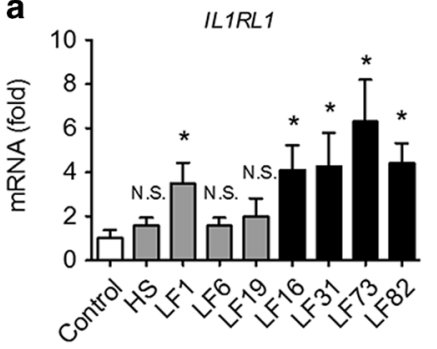

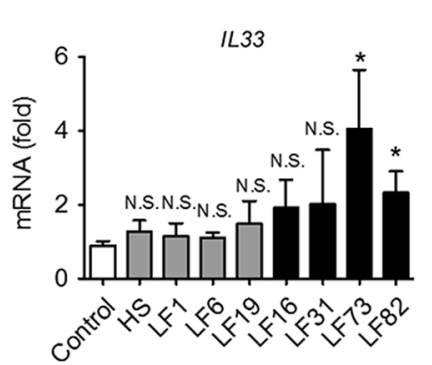

b

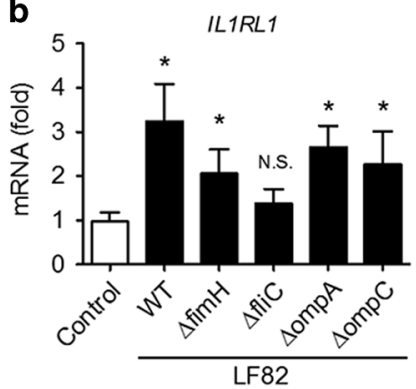

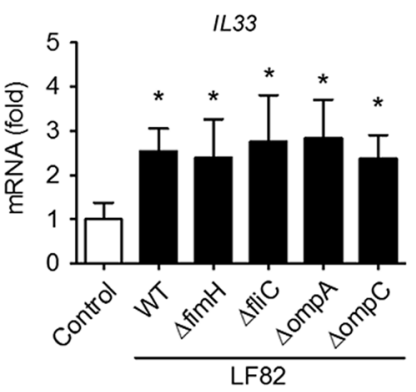
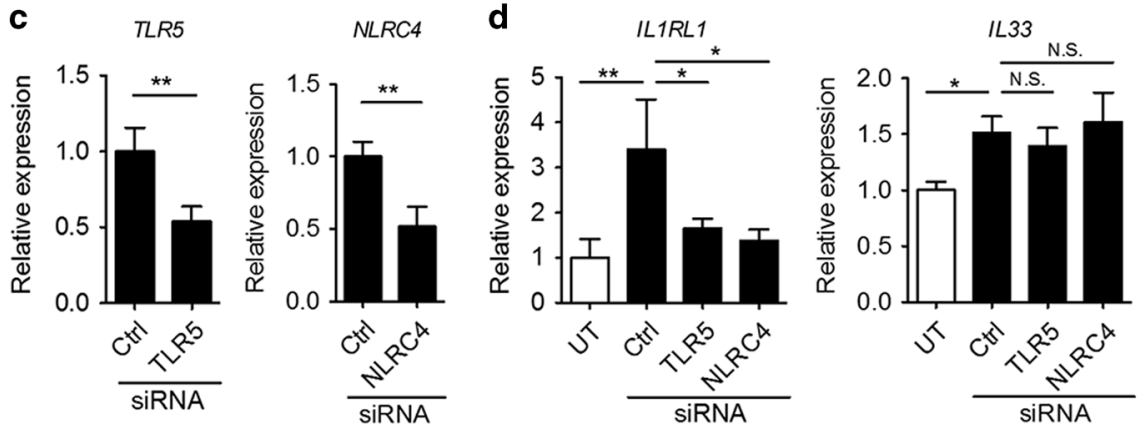

Fig. 7 The induction of ST2 by AIEC is mediated by flagellin. a T84 cells were stimulated with human commensal E. coli strain HS or E. coli strains isolated from CD patients. Gray bars indicate non-AIEC strains and black bars indicate AIEC strains based on adhesion/invasion assay. $\mathbf{b}$ T84 cells were stimulated with AIEC LF82 or various mutants of LF82 at MOI $=10$ for $3 \mathrm{~h}$ followed by $15 \mathrm{~h}$ of additional culture in the presence of gentamycin $(100 \mu \mathrm{g} / \mathrm{ml})$ to prevent bacterial overgrowth. mRNA expression of IL-33 (IL33) and ST2 (IL1RL1) was quantified by qPCR. Data shown are mean $\pm \mathrm{SD}(N=6)$. N.S.; not significant, ${ }^{*} ; P<0.05$ by Dunnett test (vs control). c TLR5 and NLRC4 mRNA were knocked-down in T84 cells using siRNA specific to human TLR5 or NLRC4. Relative expression to Ctrl Non-targeting pool siRNA are shown. Data shown are mean \pm SD $(N=3)$. ${ }^{* *} ; P<0.01$ by Student- $t$ test. d TLR5 and NLRC4 mRNA were knocked-down in T84 cells using siRNA. Cells were then stimulated with AIEC LF82 at MOI $=10$ or medium (untreated; UT) for $3 \mathrm{~h}$ followed by $15 \mathrm{~h}$ of additional culture in the presence of gentamycin (100 $\mu \mathrm{g} / \mathrm{ml}$ ) to prevent bacterial overgrowth. mRNA expression of ST2 (IL1RL1) and IL-33 (IL33) was quantified by qPCR. Data shown are mean \pm SD ( $N=3$ ). N. S.; not significant, ${ }^{*} ; P<0.05,{ }^{* *} ; P<0.01$ by One-Way ANOVA with Bonferroni post-hoc test

have flagellin. In this regard, it has been recently shown that AIEC LF82, unlike commensal $E$. coli HS, produces flagellin when exposed to intestinal mucus. ${ }^{37}$ Thus, it is conceivable that AIEC produces more flagellin than commensal $E$. coli and this hypothesis would explain why only AIEC elicits flagellinmediated pro-fibrotic signaling and subsequent development of intestinal fibrosis. Related to this notion, it also remains unclear why infection with the SPI-2 Salmonella mutant per se fails to induce fibrosis, since it also harbors flagellin. A simple explanation is that colonization of the mutant Salmonella peaked on day 2 post infection and was dramatically eradicated in the later phase of infection. On the other hand, AIEC colonization persisted longer and its abundance in the later phase of infection was higher than that of Salmonella. Thus, persistent colonization by a flagellated pathobiont resulted in intestinal fibrosis. Another possibility may be related to the capacity of the bacterium to survive intracellularly. It has been reported that SPI-2 is essential for the survival of the pathogen in phagocytic cells, such as macrophages. ${ }^{39}$ Since AIEC can survive within infected macrophages, ${ }^{8}$ it is possible that intracellular survival of pathobionts within intestinal macrophages contributes to pro-fibrotic events induced by IL-33-ST2 signaling. However, it is largely unclear how macrophage activation by AIEC affects ST2 induction in the colonic epithelium. Thus, further study is required to fully understand the mechanisms by which CD-associated pathobionts induce intestinal fibrosis.

Similarly, the extent to which other bacteria, in addition to AIEC, contribute to susceptibility to intestinal fibrosis in CD patients remains uncertain. In this regard, it has been repeatedly reported that flagellated bacteria are enriched and the luminal flagellin load is elevated in $C D$ patients compared to normal controls. ${ }^{7,40}$ Thus, dysbiotic microbiotas rich in flagellated bacteria are able to elicit intestinal fibrosis regardless of the abundance of AIEC. Consistently, colonization of germ-free mice with the gut microbiotas isolated from CD patients leads to ST2 expression in the colon. ${ }^{7}$ Thus, CD-associated pathobionts, including AIEC, contribute to the development of intestinal fibrosis via production of flagellin.

Taken together, we have unveiled a novel link between gut dysbiosis and the risk for intestinal fibrosis. Treatment of gut dysbiosis by antibiotics, probiotics, or fecal microbiota transplantation to eradicate AIEC and/or other flagellated pathobionts could be a novel therapeutic approach used to prevent intestinal fibrosis in CD. Alternatively, blockade of IL-33-ST2 signaling has the potential to be a treatment option for fibrosis in the context of $\mathrm{CD}$ patients that does not perturb the gut microbiota.

\section{MATERIALS AND METHODS}

Animals

Eight to ten-week-old SPF wild-type C57BL/6 mice were originally purchased from Jackson Laboratories. Mice were then bred and maintained in a SPF animal facility at the University of Michigan. All animal studies were performed in accordance with protocols approved by the Institutional Animal Care and Use Committee (IACUC) at the University of Michigan.

\section{Bacterial strains}

Kanamycin- and streptomycin-resistant Salmonella enterica serovar Typhimurium (S. Typhimurium) SL1344 $\Delta s p i A^{41}$ was used in mouse infection experiments. Escherichia coli strains used in this study were commensal $E$. coli strain HS, ${ }^{14}$ AIEC strains LF82, LF16, LF31, and LF73 isolated from CD patients, ${ }^{8}$ and non-AIEC-type strains LF1, LF6 and LF19 isolated from CD patients. Mutant 
a

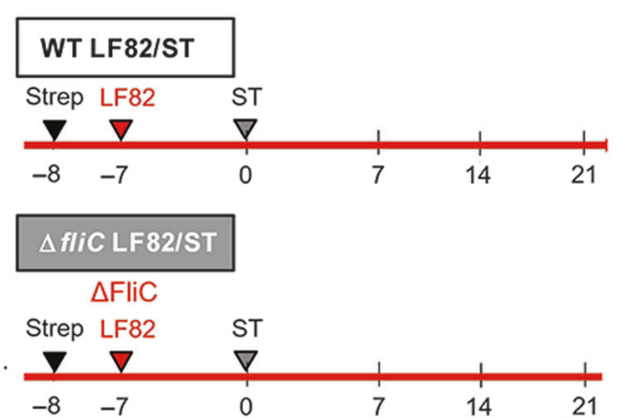

b

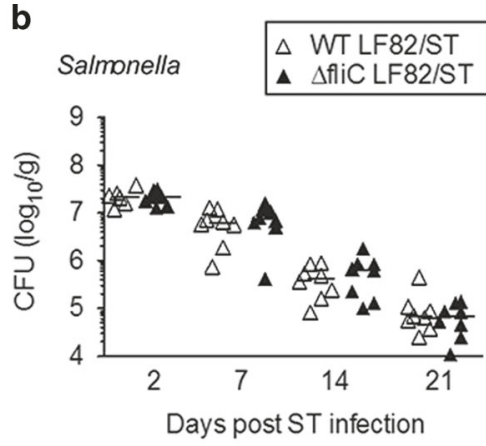

C

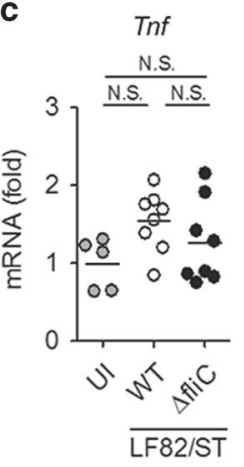

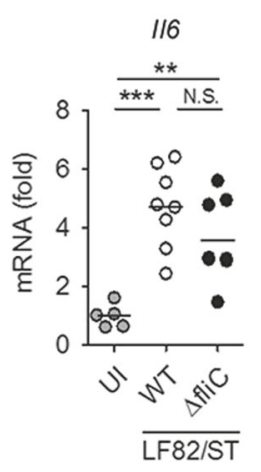

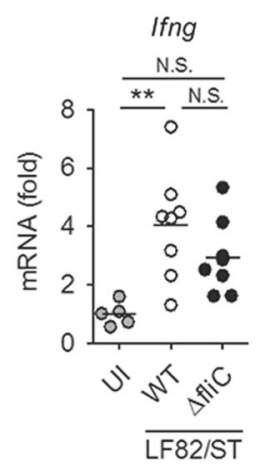

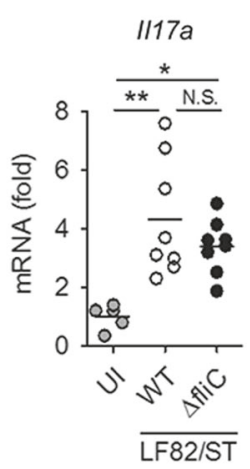

d
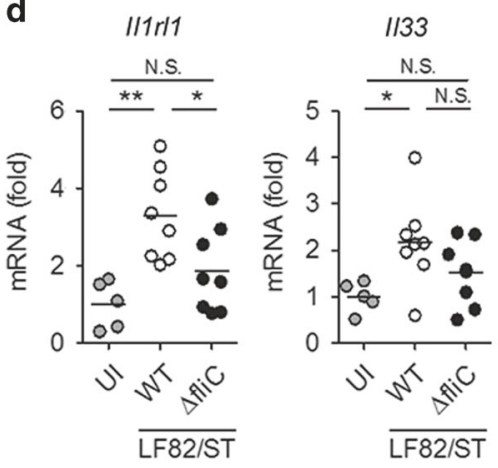

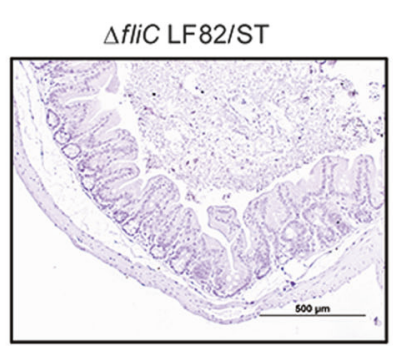

f

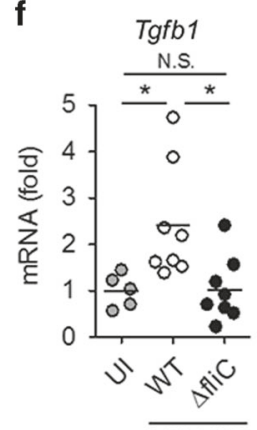

LF82/ST

h
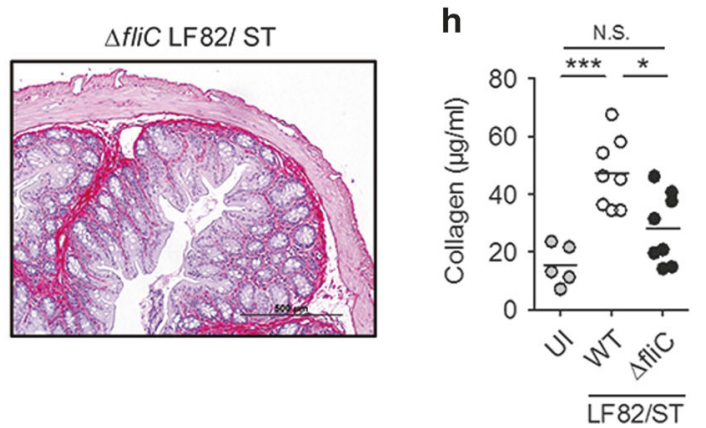

g

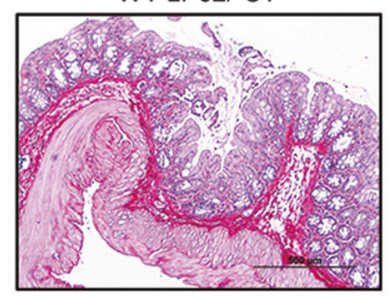

Fig. 8 Flagellin is required to promote intestinal fibrosis by a CD-associated $E$. coli. a SPF C57BL/6 mice were pre-treated with 20 mg of streptomycin (Strep) 1 day prior to E. coli inoculation. A CD-associated AIEC strain LF82 (WT or $\Delta$ fliC mutant) strains $\left(1 \times 10^{9} \mathrm{CFU}\right)$ were then inoculated to Strep-treated mice. Seven days after AIEC colonization, mice were infected with $2 \times 10^{7}$ CFU of Salmonella typhimurium $\Delta$ spiA (ST). b Intestinal colonization (in feces) of ST and AIEC LF82 (WT or $\Delta$ fliC mutant) at indicated days post ST infection. Dots represent individual mice. Bars represent median. c Expression of inflammation-related genes in the colonic mucosa (day 21 ST). d The mRNA expression of IL-33 (II33) and its receptor ST2 (I/1r/1) in the colonic mucosa. (e) Representative images of ST2 immunohistochemistry (day 21). (f) Expression of fibrosis-related genes in the colonic mucosa (day $21 \mathrm{ST}) . \mathbf{g}$, $\mathbf{h}$ Accumulation of collagen was examined by Sirius red staining (g) and collagen assay (h). Dots represent individual mice. Bars represent mean. N.S.; not significant, ${ }^{*} ; P<0.05,{ }^{* *} ; P<0.01,{ }^{* * *} ; P<0.001$ by One-Way ANOVA with Bonferroni post-hoc test 
642

LF82 strains $\Delta$ fimH, $\triangle$ flic, $\triangle o m p A$, and $\triangle o m p C$ were also used. All bacteria were grown in Luria-Bertani (LB) broth at $37{ }^{\circ} \mathrm{C}$ with shaking (180 rpm).

Salmonella infection and $E$. coli colonization

Mice were pre-treated with $20 \mathrm{mg} / 100 \mu \mathrm{l} /$ mouse of streptomycin 1 day prior to $E$. coli inoculation. Strep-treated mice were then inoculated with human $E$. coli strain HS or LF82 $\left(1 \times 10^{9}\right.$ CFU). Seven days after $E$. coli colonization, mice were infected with $2 \times$ $10^{7}$ CFU of S. Typhimurium SL1344 $\triangle$ spiA. To determine bacterial numbers in the feces, fecal pellets were collected from individual mice, homogenized in cold PBS, and plated at serial dilutions on MacConkey agar plates containing $50 \mu \mathrm{g} / \mathrm{ml}$ Strep or ampicillin (Amp). CFU counts were determined after overnight incubation at $37{ }^{\circ} \mathrm{C}$. IL-33-ST2 signaling in vivo was blocked by the intraperitoneal administration of $50 \mu \mathrm{g}$ of an anti-mouse ST2 antibody or control rat IgG (Genentech. Inc) 3 times per week. 21 days following Salmonella infection, colonic tissues were harvested and fixed in buffered 4\% paraformaldehyde. Fixed tissues were then embedded in paraffin, sectioned, and stained with heamatoxylin and eosin or picro-sirius red (Picrosciences, Inc.).

Quantitative real-time PCR

RNA was extracted from colonic tissues using the E.Z.N.A. ${ }^{\circledR}$ Total RNA Kit I (Omega Bio-tek). Reverse transcription was performed using the High-Capacity cDNA Reverse Transcription Kit (Thermo Fisher Scientific). Quantitative PCR was performed with SYBR Green qPCR Kits (Radiant molecular tolls). The cycling conditions were $95^{\circ} \mathrm{C}$ for $3 \mathrm{~min}$ and then 39 cycles of $95^{\circ} \mathrm{C}$ for $15 \mathrm{~s}, 60^{\circ} \mathrm{C}$ for $1 \mathrm{~min}$. The relative mRNA expression of target gene was normalized against that of the $\beta$-actin gene in the same RNA preparation. The following primer sets were used for amplifications: Human h/L1RL1-Fw; 5'-CTG TCT GGC CCT GAA TाT GC-3', Human IL1RL1-Rv; 5'-AGC AGA GTG GCC TCA ATC CA-3', Human IL33-Fw; 5'-ATC CCA ACA GAA GGC CAA AG-3', Human IL33-Rv; 5'CCA AAG GCA AAG CAC TCC AC-3', Human TGFB1-Fw; 5'-CTA ATG GTG GAA ACC CAC AAC G-3', Human TGFB1-Rv; 5'-TAT CGC CAG GAA TTG TTG CTG-3', Human COL1A2-Fw; 5'-TCT GGA TGG ATT GAA GGG ACA-3', Human COL1A2-Rv; 5'-CCA ACA CGT CCT CTC TCA CC-3', Human TNF-Fw; 5'-GCC TCT TCT CAT TCC TGC TT-3', Human TNF-Rv; 5'-TGG GAA CTT CTC ATC CCT TTG-3', Human TLR5-Fw; 5'- TGC CTT GAA GCC TTC AGT TAT G-3', Human TLR5-Rv; 5'- CCA ACC ACC ACC ATG ATG AG-3', Human NLRC4-Fw; 5'- ACG TGC TAG AAC AGC TCA CC-3', Human NLRC4-Rv; 5'-AGC TTG ACG AGT TGT GGG AC-3', Human ACTB-Fw; 5'-CTC TTC CAG CCT TCC TTC CTG-3', Human ACTB-Rv; 5'-GAA GCA TाT GCG GTG GAC GAT3'. Mouse $/ 11 \mathrm{r} / 1-\mathrm{Fw} ; 5^{\prime}$-AGA CCT GTT ACC TGG GCA AG-3', Mouse //1r/1-Rv; 5'-CAC CTG TCT TCT GCT ATT CTG G-3', Mouse I/33-Fw; 5'TCC TाG CTT GGC AGT ATC CA-3', Mouse $/ 133$-Rv; 5'-TGC TCA ATG TGT CAA CAG ACG-3', Mouse Tgfb1-Fw; 5'-CTC CCG TGG CTT CTA GTG C-3', Mouse Tgfb1-Rv; 5'-GCC TTA GTT TGG ACA GGA TCT G -3', Mouse Col1a2-Fw; 5'-TGG ATA CGC GGA CTC TGT TG-3', Mouse Col1a2-Rv; 5'-CCC TाT CGT ACT GAT CCC GAT T-3', Mouse Tnf-Fw; 5'-GCC TCC CTC TCA TCA GTT CT-3', Mouse Tnf-Rv; 5'-CAC TTG GTG GTT TGC TAC GA-3', Mouse I/6-Fw; 5'-GAG GAT ACC ACT CCC AAC AGA CC-3', Mouse Il6-Rv; 5'-AAG TGC ATC ATC GTT GTT CAT ACA3', Mouse Ifng-Fw; 5'-ATC TGG AGG AAC TGG CAA AA-3', Mouse Ifng-Rv; 5'-TTC AAG ACT TCA AAG AGT CTG AGG TA -3', Mouse I/17a-Fw; 5'-CAG GGA GAG CTा CAT CTG TGT-3', Mouse //17a-Rv; 5'GCT GAG CTT TGA GGG ATG AT-3', Mouse Actb-Fw; 5'-AAG TGT GAC GTT GAC ATC CG-3', Mouse Actb-Rv; 5'-GAT CCA CAT CTG CTG GAA GG-3'.

\section{Collagen assay}

Colonic specimens were homogenized in $2.5 \mathrm{M}$ acetic acid containing $0.1 \mathrm{mg} / \mathrm{ml}$ pepsin. The samples were then dried overnight at $37{ }^{\circ} \mathrm{C}$. Total soluble collagen was quantified by Soluble Collagen Assay (Cell Biolabs, Inc).
Cell culture

Human primary colonic fibroblasts were isolated from disease-free surgical margins found in surgically resected colonic specimens obtained from a diverticulitis patient. Human fibroblasts were then cultured in Dulbecco's Modified Eagle's Medium (DMEM) supplemented with $10 \%$ fetal bovine serum (FBS) and antibiotics (penicillin/streptomycin). Human colon carcinoma cells (T84) were grown in Ham's F12 medium + DMEM (1:1) supplemented with $10 \%$ FBS and antibiotics. Cells $\left(1 \times 10^{5}\right.$ cells/500 $\mu$ l /well; 24 well plate) were stimulated with $E$. coli at a $\mathrm{MOI}=10$ for $3 \mathrm{~h}$ followed by $15 \mathrm{~h}$ of additional culture in the presence of gentamycin (100 $\mu \mathrm{g} / \mathrm{ml}$ ) to prevent bacterial overgrowth.

Knock-down of flagellin receptors

SMARTpool siRNA for human TLR5 and NLRC4 were purchased from Dharmacon. The siRNAs or Non-targeting control siRNA (Dharmacon) were transfected to T84 cells using Lipofectamine RNAiMAX (Life Technologies) according to the manufacturers' instructions. Two days post-transfection, the cells were stimulated with $E$. coli $(\mathrm{MOI}=10)$ for $3 \mathrm{~h}$ followed by $15 \mathrm{~h}$ of additional culture in the presence of gentamycin $(100 \mu \mathrm{g} / \mathrm{ml})$.

Immunohistochemistry

The colonic tissues were harvested, fixed with $4 \%$ paraformaldehyde and embedded in paraffin. The tissue samples were then cut into $4 \mu \mathrm{m}$ thick sections. Rehydrated paraffin sections were boiled in a microwave oven for epitope retrieval using a sodium citrate buffer ( $\mathrm{pH} \mathrm{6,10} \mathrm{min).} \mathrm{Slides} \mathrm{were} \mathrm{stained} \mathrm{with} \mathrm{an} \mathrm{anti-}$ mouse ST2 antibody (Abcam, ab25877, 1:500 dilution) overnight at $4{ }^{\circ} \mathrm{C}$ and then incubated with a rabbit HRP polyclonal antibody for $1 \mathrm{~h}$ at room temperature. HRP was then visualized with DAB (VECTOR).

\section{Statistical analyses}

Statistical analyses were performed using GraphPad Prism software version 5.0 (GraphPad Software, Inc, San Diego, CA). Differences between 2 groups were evaluated using Student's $t$ test (parametric) or the Mann-Whitney $U$-test (nonparametric). For comparisons of more than 3 groups, statistical analysis was performed using One-way analysis of variance (parametric) or the Kruskal-Wallis test (nonparametric), followed by the Bonferroni correction for parametric samples, or the Dunn test for nonparametric samples as a post hoc test. $P$-values less than 0.05 were considered significant.

\section{ACKNOWLEDGEMENTS}

The authors thank the University of Michigan Center for Gastrointestinal Research (UMCGR) (NIH 5P30DK034933) for technical assistance. This work was supported by Kenneth Rainin Foundation Innovator Award (N.K.), National Institute of Health DK110146 and DK108901 (N.K.), T32 DK094775 (T.L.M.), Crohn's and Colitis Foundation of America (N. K. and H. N.-K.), Mitsukoshi Health and Welfare Foundation (J.I.), JSPS Postdoctoral Fellowship for Research Abroad (S.K. and H. N.-K.), the Uehara Memorial Foundation Postdoctoral Fellowship Award (S.K. and K.S.). Clinical and Translational Science Awards (CTSA) Program (S.K.), and Prevent Cancer Foundation (S.K.). All data necessary to reproduce the results presented herein are available in the main text.

\section{AUTHOR CONTRIBUTIONS}

J.I. and N.K. conceived and designed experiments. J.I. conducted most of the experiments with help from S.K., K.S., H.N.-K., A.H., T.L.M. and P.K.. P.D.R.H. and N.B. provided advice and discussion. N.B. provided critical materials. J.I., S.K., K.S. and N.K. analyzed the data. J.I. and N.K. wrote the manuscript with contributions from all authors.

ADDITIONAL INFORMATION

Competing interests: The authors declare no competing interests. 
Publisher's note: Springer Nature remains neutral with regard to jurisdictional claims in published maps and institutional affiliations.

\section{REFERENCES}

1. Xavier, R. J. \& Podolsky, D. K. Unravelling the pathogenesis of inflammatory bowel disease. Nature 448, 427-434 (2007).

2. Ananthakrishnan, A. N. Epidemiology and risk factors for IBD. Nat. Rev. Gastroenterol. Hepatol. 12, 205-217 (2015).

3. Rieder, F., Fiocchi, C. \& Rogler, G. Mechanisms, management, and treatment of fibrosis in patients with inflammatory bowel diseases. Gastroenterology 152, 340-350. e346 (2017).

4. Latella, G., Di Gregorio, J., Flati, V., Rieder, F. \& Lawrance, I. C. Mechanisms of initiation and progression of intestinal fibrosis in IBD. Scand. J. Gastroenterol. 50, 53-65 (2015)

5. Sartor, R. B. \& Wu, G. D. Roles for intestinal bacteria, viruses, and fungi in pathogenesis of inflammatory bowel diseases and therapeutic approaches. Gastroenterology 152, 327-339. e324 (2017).

6. Kamada, N., Seo, S. U., Chen, G. Y. \& Nunez, G. Role of the gut microbiota in immunity and inflammatory disease. Nat. Rev. Immunol. 13, 321-335 (2013).

7. Nagao-Kitamoto, $\mathrm{H}$. et al. Functional characterization of inflammatory bowel disease-associated gut dydbiosis in gnotobiotic mice. Cell Mol. Gastroenterol. Hepatol. 2, 468-481 (2016).

8. Darfeuille-Michaud, A. et al. High prevalence of adherent-invasive Escherichia coli associated with ileal mucosa in Crohn's disease. Gastroenterology 127, 412-421 (2004)

9. Small, C. L., Reid-Yu, S. A., McPhee, J. B. \& Coombes, B. K. Persistent infection with Crohn's disease-associated adherent-invasive Escherichia coli leads to chronic inflammation and intestinal fibrosis. Nat. Commun. 4, 1957 (2013).

10. Carvalho, F. A. et al. Crohn's disease-associated Escherichia coli LF82 aggravates colitis in injured mouse colon via signaling by flagellin. Inflamm. Bowel Dis. 14, 1051-1060 (2008).

11. Small, C. L., Xing, L., McPhee, J. B., Law, H. T. \& Coombes, B. K. Acute infectious gastroenteritis potentiates a Crohn's disease pathobiont to fuel ongoing inflammation in the post-infectious period. PLoS. Pathog. 12, e1005907 (2016).

12. Rieder, F., Kessler, S., Sans, M. \& Fiocchi, C. Animal models of intestinal fibrosis: new tools for the understanding of pathogenesis and therapy of human disease. Am. J. Physiol. Gastrointest. Liver Physiol. 303, G786-G801 (2012).

13. Grassl, G. A., Valdez, Y., Bergstrom, K. S., Vallance, B. A. \& Finlay, B. B. Chronic enteric salmonella infection in mice leads to severe and persistent intestinal fibrosis. Gastroenterology 134, 768-780 (2008).

14. Rasko, D. A. et al. The pangenome structure of Escherichia coli: comparative genomic analysis of E. coli commensal and pathogenic isolates. J. Bacteriol. 190, 6881-6893 (2008).

15. Schmitz, J. et al. IL-33, an interleukin-1-like cytokine that signals via the IL-1 receptor-related protein ST2 and induces T helper type 2-associated cytokines. Immunity 23, 479-490 (2005).

16. Fichtner-Feigl, S. et al. Induction of IL-13 triggers TGF-beta1-dependent tissue fibrosis in chronic 2,4,6-trinitrobenzene sulfonic acid colitis. J. Immunol. 178, 5859-5870 (2007).

17. Vancamelbeke, M. et al. Genetic and transcriptomic bases of intestinal epithelia barrier dysfunction in inflammatory bowel disease. Inflamm. Bowel Dis. 23 1718-1729 (2017).

18. Sedhom, M. A. et al. Neutralisation of the interleukin-33/ST2 pathway ameliorates experimental colitis through enhancement of mucosal healing in mice. Gut $\mathbf{6 2}$, 1714-1723 (2013).
19. Moussion, C., Ortega, N. \& Girard, J. P. The IL-1-like cytokine IL-33 is constitutively expressed in the nucleus of endothelial cells and epithelial cells in vivo: a novel 'alarmin'? PLOS. ONE. 3, e3331 (2008).

20. Dreux, N. et al. Point mutations in FimH adhesin of Crohn's disease-associated adherent-invasive Escherichia coli enhance intestinal inflammatory response. PLoS. Pathog. 9, e1003141 (2013).

21. Barnich, N., Boudeau, J., Claret, L. \& Darfeuille-Michaud, A. Regulatory and functional co-operation of flagella and type 1 pili in adhesive and invasive abilities of AIEC strain LF82 isolated from a patient with Crohn's disease. Mol. Microbiol. 48 781-794 (2003).

22. Rolhion, N., Carvalho, F. A. \& Darfeuille-Michaud, A. OmpC and the sigma(E) regulatory pathway are involved in adhesion and invasion of the Crohn's diseaseassociated Escherichia coli strain LF82. Mol. Microbiol. 63, 1684-1700 (2007).

23. Palmela, C. et al. Adherent-invasive Escherichia coli in inflammatory bowel disease. Gut 67, 574-587 (2018)

24. Winter, S. E. et al. Host-derived nitrate boosts growth of E. coli in the inflamed gut. Science 339, 708-711 (2013).

25. Kamada, N., Chen, G. Y., Inohara, N. \& Nunez, G. Control of pathogens and pathobionts by the gut microbiota. Nat. Immunol. 14, 685-690 (2013).

26. McHedlidze, T. et al. Interleukin-33-dependent innate lymphoid cells mediate hepatic fibrosis. Immunity 39, 357-371 (2013).

27. Koca, S. S. et al. The IL-33 gene is related to increased susceptibility to systemic sclerosis. Rheumatol. Int. 36, 579-584 (2016).

28. Wu L. et al. IL-33 Can Promote the Process of Pulmonary Fibrosis by Inducing the Imbalance Between MMP-9 and TIMP-1. Inflammation. 41, 878-885 (2018).

29. Pushparaj, P. N. et al. Interleukin-33 exacerbates acute colitis via interleukin-4 in mice. Immunology 140, 70-77 (2013).

30. Beltran, C. J. et al. Characterization of the novel ST2/IL-33 system in patients with inflammatory bowel disease. Inflamm. Bowel Dis. 16, 1097-1107 (2010).

31. Pastorelli, L. et al. Epithelial-derived IL-33 and its receptor ST2 are dysregulated in ulcerative colitis and in experimental Th1/Th2 driven enteritis. Proc. Natl Acad. Sci. USA 107, 8017-8022 (2010).

32. Seo, D. H. et al. Interleukin-33 regulates intestinal inflammation by modulating macrophages in inflammatory bowel disease. Sci. Rep. 7, 851 (2017).

33. Mindt, B. C., Fritz, J. H. \& Duerr, C. U. Group 2 Innate Lymphoid Cells in Pulmonary Immunity and Tissue Homeostasis. Front. Immunol. 9, 840 (2018).

34. Lo B. C. et al. The orphan nuclear receptor ROR alpha and group 3 innate lymphoid cells drive fibrosis in a mouse model of Crohn's disease. Science immunology. 1, eaaf8864 (2016).

35. Denney, L. et al. Pulmonary epithelial cell-derived cytokine TGF-beta1 Is a critical cofactor for enhanced innate lymphoid cell function. Immunity 43, 945-958 (2015).

36. Son, A. et al. TWEAK/Fn14 pathway promotes a T helper 2-type chronic colitis with fibrosis in mice. Mucosal Immunol. 6, 1131-1142 (2013).

37. Sevrin G. et al. Adaptation of adherent-invasive E. coli to gut environment: Impact on flagellum expression and bacterial colonization ability. Gut Microbes. 1-17 (2018). https://doi.org/10.1080/19490976.2017.1421886.

38. Chassaing, B., Koren, O., Carvalho, F. A., Ley, R. E. \& Gewirtz, A. T. AIEC pathobiont instigates chronic colitis in susceptible hosts by altering microbiota composition. Gut 63, 1069-1080 (2014).

39. Buckner, M. M., Croxen, M. A., Arena, E. T. \& Finlay, B. B. A comprehensive study of the contribution of Salmonella enterica serovar Typhimurium SPI2 effectors to bacterial colonization, survival, and replication in typhoid fever, macrophage, and epithelial cell infection models. Virulence 2, 208-216 (2011).

40. Duck, L. W. et al. Isolation of flagellated bacteria implicated in Crohn's disease. Inflamm. Bowel Dis. 13, 1191-1201 (2007).

41. Hensel, M. et al. Simultaneous identification of bacterial virulence genes by negative selection. Science 269, 400-403 (1995). 